\title{
Forecasting of Indian Stock Market Index S\&P CNX Nifty 50 Using Artificial Intelligence
}

\author{
Dr. Jay Desai ${ }^{1}$, Nisarg A Joshi ${ }^{2}$ \\ ${ }^{1,2}$ Shri Chimanbhai Patel Institute of Management and Research, Ahmedabad, Gujarat, India
}

\begin{abstract}
This paper presents a computational approach for predicting the S\&P CNX Nifty 50 Index. A neural network based model has been used in predicting the direction of the movement of the closing value trend of the index by predicting the seven day simple moving average value change after seven days. The model presented in the paper also confirms that it can be used to predict price index value trend of the stock market. After studying the various features of the network model, a suitable model for stocks forecast is proposed. The model has used the preprocessed data set of closing value of S\&P CNX Nifty 50 Index. The data set encompassed the trading days from $1^{\text {st }}$ September, 2013 to $30^{\text {th }}$ April, 2015. In the study the model has been tested to forecast the stock market trend for a period of five months. Accuracy of the performance of the neural network is compared with buy and hold returns of the index. The model generated return of $70.42 \%$ against buy and hold return of $47.41 \%$.
\end{abstract}

Keywords: artificial intelligence, neural network, S\&P, index, forecasting

\section{Introduction}

Recently forecasting stock market return is gaining more attention, maybe because of the fact that if the direction of the market is successfully predicted the investors may be better guided. The profitability of investing and trading in the stock market to a large extent depends on the predictability. If any system be developed which can consistently predict the trends of the dynamic stock market, would make the owner of the system wealthy. More over the predicted trends of the market will help the regulators of the market in making corrective measures.

Another motivation for research in this field is that it possesses many theoretical and experimental challenges. The most important of these is the Efficient Market Hypothesis (EMH); see Eugene Fama's(1970) "Efficient Capital Markets". The hypothesis says that in an efficient market, stock market prices fully reflect available information about the market and its constituents and thus any opportunity of earning excess profit ceases to exist. So it is ascertain that no system is expected to outperform the market predictably and consistently. Hence, modeling any market under the assumption of EMH is only possible on the speculative, stochastic component not on the changes on the changes in value or other fundamental factors (Pan Heping., 2004). Another related theory to EMH is the Random Walk Theory, which states that all future prices do not follow any trend or pattern and are random departure from the previous prices. There has been a lot of debate about the validity of the EMH and random walk theory. However with the advent of computational and intelligent finance, and behavioral finance, economists have tried to establish an opposite hypothesis which may be collectively called as the Inefficient Market Hypothesis (IMH). IMH states that financial markets are at least not always efficient, the market is not always in a random walk, and inefficiencies exists. (Pan Heping., 2003). The origins of disparity of assumptions of EMH go back to the work of Mandelbrot (1960), when he studied the cotton prices in New York exchange. In his studies on the cotton prices he found that the data did not fit the normal distribution but instead produced symmetry from the point of view of scaling. The sequences of changes are independent of scaling; curves of daily changes and the curves of monthly change matched perfectly. Mandelbrot presented the fractals of the financial markets. Subsequently, with evolution in this field of research Pan Heping in 2003 postulated the Swing Market Hypothesis (SMH) which states that market is sometimes efficient and sometimes inefficient; and the tends to swing between these two modes intermittently. The theory also proposes that the market movement can be decomposed into four types of components: dynamical swings, physical cycles, abrupt momentums and random walks. (Pan Heping)

Moreover, many researchers claim that the stock market is a chaos system. Chaos is a nonlinear deterministic system which only appears random because of its irregular fluctuations. These systems are highly sensitive to the initial conditions of the systems. These systems are dynamic, a periodic, and complicated and are difficult to deal with normal analytical methods. The neural networks are effective in learning such nonlinear chaotic systems because they make very few assumptions about the functional form of the underlying dynamic dependencies and their initial conditions. This may eventually question the traditional financial theory of efficient market.

Many researchers and practitioners have proposed many models using various fundamental, technical and analytical techniques to give a more or less exact prediction. Fundamental analysis involves the in-depth analysis of the changes of the stock prices in terms of exogenous macroeconomic variables. It assumes that the share price of a stock depends on its intrinsic value and the expected return of the investors. But this expected return is subjected to change as new information pertaining to the stock is available in the market which in turn changes the share price. Moreover, the analysis of the economic factors is quite subjective as the interpretation totally lays on the 


\section{International Journal of Science and Research (IJSR) \\ ISSN (Online): 2319-7064 \\ Index Copernicus Value (2013): 6.14 | Impact Factor (2014): 5.611}

intellectuality of the analyst. Alternatively, technical analysis centers on using price, volume, and open interest statistical charts to predict future stock movements. The premise behind technical analysis is that all of the internal and external factors that affect a market at any given point of time are already factored into that market's price. (Louis. B. Mendelsohn, 2000).

Apart from these commonly used methods of prediction, some traditional time series forecasting tools are also used for the same. In time series forecasting, the past data of the prediction variable is analyzed and modeled to capture the patterns of the historic changes in the variable. These models are then used to forecast the future prices.

There are mainly two approaches of time series modeling and forecasting: linear approach and the nonlinear approach. Mostly used linear methods are moving average, exponential smoothing, time series regression etc. One of the most common and popular linear method is the Autoregressive integrated moving average (ARIMA) model (Box and Jenkins (1976)). It presumes linear model but is quite flexible as it can represent different types of time series, i.e. Autoregressive (AR), moving average (MA) and combined AR and MA (ARMA) series.

However, there is not much evidence that the stock market returns are perfectly linear for the very reason that the residual variance between the predicted return and the actual is quite high. The existence of the nonlinearity of the financial market is propounded by many researchers and financial analyst. (Abhyankar, 1997). Some parametric nonlinear model such as Autoregressive Conditional Heteroskedasticity and General Autoregressive Conditional Heteroskedasticity has been in use for financial forecasting. But most of the nonlinear statistical techniques require that the nonlinear model must be specified before the estimation of the parameters is done.

During last few years there has been much advancement in the application of neural network in stock market indices forecasting with a hope that market patterns can be extracted. The novelty of the ANN lies in their ability to discover nonlinear relationship in the input data set without a priori assumption of the knowledge of relation between the input and the output. (Hagen et al., 1996). they independently learn the relationship inherent in the variables. From statistical point of view neural networks are analogous to nonparametric, nonlinear, regression model. So, neural network suits better than other models in predicting the stock market returns.

A neural network is a massively parallel distributed processor made up of simple processing unit which has a natural propensity for storing experiential knowledge and making it available for use. (Simon Haykin, (1999). Neural networks have remarkable ability to derive meaning from complicated or imprecise data. They are used to extract patterns and detect trends that are too complex to be noticed by either humans or other computer techniques. From statistical inference neural networks are analogous to nonparametric, nonlinear, regression model. However, the traditional statistical models have limitations in understanding the relationship between the input and the output of the system because of the complex and chaos nature of the system.

There are several distinguished features that propound the use of neural network as a preferred tool over other traditional models of forecasting.

Neural networks are nonlinear in nature and where most of the natural real world systems are nonlinear in nature, neural networks are preferred over the traditional linear models. This is because the linear models generally fail to understand the data pattern and analyze when the underlying system is a nonlinear one. However, some parametric nonlinear model such as Autoregressive Conditional Heteroskedasticity (Engle, 1982) and General Autoregressive Conditional Heteroskedasticity have been in use for financial forecasting. But most of the nonlinear statistical techniques require that the non-l inear model must be specified before the estimation of the parameters is done and generally it happens that pre-specified nonlinear models may fail to observe the critical features of the complex system under study.

Neural networks are data driven models. The novelty of the neural network lies in their ability to discover nonlinear relationship in the input data set without a priori assumption of the knowledge of relation between the input and the output (Hagen et al., 1996) the input variables are mapped to the output variables by squashing or transforming by a special function known as activation function. They independently learn the relationship inherent in the variables from a set of labeled training example and therefore involves in modification of the network parameters.

Neural Networks have a built in capability to adapt the network parameters to the changes in the studied system. A neural network trained to a particular input data set corresponding to a particular environment; can be easily retrained to a new environment to predict at the same level of environment. Moreover, when the system under study is non stationary and dynamic in nature, the neural network can change its network parameters (synaptic weights) in real time.

So, neural network suits better than other models in predicting the stock market returns.

\section{Literature Review}

In the last two decades forecasting of stock returns has become an important field of research. In most of the cases the researchers had attempted to establish a linear relationship between the input macroeconomic variables and the stock returns. But with the discovery of nonlinearity in the stock market index returns (A. Abhyankar et al. 1997), there has been a great shift in the focus of the researchers towards the nonlinear prediction of the stock returns. Although, there after many literatures have come up in nonlinear statistical modeling of the stock returns, most of them required that the nonlinear model be specified before the estimation is done. But for

\section{Volume 4 Issue 11, November 2015}




\section{International Journal of Science and Research (IJSR) \\ ISSN (Online): 2319-7064 \\ Index Copernicus Value (2013): 6.14 | Impact Factor (2014): 5.611}

the reason that the stock market return being noisy, uncertain, chaotic and nonlinear in nature, ANN has evolved out to be better technique in capturing the structural relationship between a stock's performance and its determinant factors more accurately than many other statistical techniques (Refenes et al., S.I. $\mathrm{Wu}$ et al., Schoeneburg, E.,).

In literature, different sets of input variables are used to predict stock returns. In fact, different input variables are used to predict the same set of stock return data. Some researchers used input data from a single time series where others considered the inclusion of heterogeneous market information and macro-economic variables. Some researchers even preprocessed these input data sets before feeding it to the ANN for forecasting.

Chan., Wong., and Lam., implemented a neural network model using the technical analysis variables for listed companies in Shanghai Stock Market. In this paper performance of two learning algorithm and two weight initialization methods are compared. The results reported that prediction of stock market is quite possible with both the algorithm and initialization methods but the performance of the efficiency of the back propagation can be increased by conjugate gradient learning and with multiple linear regression weight initializations.

Other prominent literatures are that of Siekmann et al. (2001) who used fuzzy rules to split inputs into increasing, stable, and decreasing trend variables. Siekmann et al. (2001) implemented a network structure that contains the adaptable fuzzy parameters in the weights of the connections between the first and second hidden layers.

Kim and Han (2000) used a genetic algorithm to transform continuous input values into discrete ones. The genetic algorithm was used to reduce the complexity of the feature space. Kishikawa and Tokinaga (2000) used a wavelet transform to extract the short-term feature of stock trends. Kim and Han (2000) used neural network modified by Genetic Algorithm. Kim and Chun (1998) used refined probabilistic NN (PNN) to predict a stock market index. Pantazopoulos et al. (1998) presented a neurofuzzy approach for predicting the prices of IBM stock.

Chenoweth, Tim., Obradovic, Zoran., used specialized neural network as preprocessing component and a decision rule base. The preprocessing component determine the most relevant features for stock market prediction, remove the noise, and separate the remaining patterns into two disjoint sets. Next, the two neural networks predict the market's rate of return, with one network trained to recognize positive and the other negative returns.

Some work has also been reported in portfolio construction, for Roman, Jovina and Jameel, Akhtar in their paper proposed a new methodology to aid in designing a portfolio of investment over multiple stock markets. For that they used back propagation and recurrent network and also the contextual market information. They developed a determinant using the accuracy of prediction of the neural network and the stock return of the previous year and used it to select the stock market among other markets.

In many papers ARIMA model has been used as a benchmark model in order to compare the forecasting accuracy of the ANN. Jung-Hua Wang; Jia-Yann Leu developed a prediction system of recurrent neural network trained by using features extracted from ARIMA analysis. Then after differencing the raw data of the TSEWSI series and then examining the autocorrelation and partial autocorrelation function plots, they identified the series as a nonlinear version of ARIMA $(1,2,1)$. Neural networks were trained by using second difference data and were seen to give better predictions than otherwise trained by using raw data. Jingtao Yao, Chew Lim Tan and HeanLee Poh developed a neural network that was used to predict the stock index of Kuala Lumpur Stock Exchange. The used trading strategies to a paper profit were recorded and were compared with that of the ARIMA model. The results showed that the performance of the neural net was better than that of the ARIMA. It was also asserted that useful prediction can be made even without the use of extensive data or knowledge.

In the work by Refenes et al, (1997), they compared the performance of back propagation network and regression models to predict the stock market returns. Desai, V. S. (1998), compared the performance of linear regression with that of the neural network in forecasting the stock returns.

Researchers have tested the accuracy of ANN in predicting the stock market index return of most developed economies across the globe. Literatures are available for forecasting index returns of U.S markets like NYSE (U.S.A.), FTSE (U.K.), DJIA (U.S.A.), S\&P500 (U.S.A.) Few papers are also available in context to Asian stock markets like Hang Seng Stock Exchange, Korea Stock Exchange Tokyo Stock Exchange and Taiwan Stock Exchange.

Some literatures are also available in Indian context. Panda, C. and Narasimhan, V. used the artificial neural network to forecast the daily returns of Bombay Stock Exchange (BSE) Sensitive Index (Sensex). They compared the performance of the neural network with performances of random walk and linear autoregressive models. They reported that neural network out-performs linear autoregressive and random walk models by all performance measures in both in-sample and out-ofsample forecasting of daily BSE Sensex returns.

In another paper, Dutta,G. et.al. Studied the efficacy of ANN in modeling the Bombay Stock Exchange (BSE) SENSEX weekly closing values. They developed two networks with inputs as the weekly closing value, 52-week moving average of the weekly closing SENSEX values, 5week moving average of the same, and the 10-week Oscillator for the past 200 weeks for one neural net. And for the other network the inputs are the weekly closing value, 52-week moving average of the weekly closing SENSEX values, 5-week moving average of the same and the 5- week volatility for the past 200 weeks. To assess the performance of the networks they used the neural networks to predict the weekly closing SENSEX values for the two-

\section{Volume 4 Issue 11, November 2015}




\section{International Journal of Science and Research (IJSR) \\ ISSN (Online): 2319-7064 \\ Index Copernicus Value (2013): 6.14 | Impact Factor (2014): 5.611}

year period beginning January 2002. The root mean square error (RMSE) and mean absolute error (MAE) are chosen as indicators of performance of the networks. The proposed network has been tested with stock data obtained from the Indian stock Market BSE Index. Bishnoi T. R., et al has analyzed the behavior of daily and weekly returns of five Indian stock market indices for random walk during April1996 to June-2001. They have tested the indices for normality, autocorrelation using Qstatistic \& Dickey-Fuller test and analyzed variance ratio using homoscedastic and heteroscedastic test estimates. The results support that Indian stock market indices do not follow random walk.

In their paper Majumdar and Hussian in the year 2010 could develop a neural net that could forecast Nifty direction on closing for the next day with $69.72 \%$ accuracy.

The previous studies have used various forecasting techniques in order to predict the stock market trends. Some attempted to forecast the daily returns where others developed forecasting models to predict the rate of returns of individual stocks. In many papers it was also found that researchers have attempted to compare their results with other statistical tools. And these findings provide strong motivation for modeling forecasting tools for stock market prediction.

\section{Data and Methodology}

The data employed in the study consists of daily closing prices of S\&P CNX Nifty 50 Index. The data set encompassed the trading days from $1^{\text {st }}$ September, 2013 to $30^{\text {th }}$ April, 2015. The reason for this time frame is beginning of a new bull market in India from the year 2013 and possibility of change in the walk of the market then the previous bullish years. The study makes an attempt to design a simple neural network model where in most of the critical issues pertaining to performance of the neural network will be addressed.

The performance of the neural network largely depends on the model of the Neural Network. Issues critical to the neural network modeling like selection of input variables, data preprocessing technique, network architecture design and performance measuring statistics, are considered carefully.

\section{Selection of input variables:}

Selection of input variable for the neural network model is a critical factor for the performance of the neural network because it contains important information about the complex nonlinear structures of the data. It also facilitates the neural network to understand the movements in the time series. The input variables selected for this model are the lagged observation of the time series being forecasted, which in this case is the closing prices of S\&P CNX Nifty 50 Index. The criticality in selecting the input variables lies in selecting the number of input variables and the lag between each. With less of lag between the inputs the correlation between the lagged variable increases which may result in an over-fitting phenomenon. On the other hand, with increase in the lag between each input variable the neural network may lose out essential information of input variables, resulting in under-learning. To handle with this dilemma of over fitting or under- learning and select an optimal structure, we have considered various lagged structure (multiple lag input variable with different lag between each) and test the performance of the neural network on a trial and error basis.

In order to evaluate the performance of the neural network it was tested on out of data sample. Separate set of data for training and testing are defined.

\section{Data Preprocessing}

The performance and the reliability of a neural network model also to a large extent depend on the quality of the data used. As neural networks are pattern recognizers, the data presented to it largely influences the accuracy of the result. The data preprocessing of the input variable of the neural network model facilitates de-trending of the data and highlight essential relationship, so as to facilitate proper network learning process.

In order to improve the performance of the network a nonlinear scaling method is adopted. A logarithmic first differencing was used to preprocess the scaled down series of closing price of the index. Logarithmic first difference takes the logarithmic value of the series and then taking the difference.

$X * k, n=\ln (X k, n)-\ln (X k,(n-1))$

Here in equation, $\mathbf{X k} \mathbf{k}, \mathbf{( n - 1 )}$ is yesterday's closing price and $\mathbf{X k}, \mathbf{n}$ is today's closing price. The output target is defined as follows, if the close of a day is $\mathrm{Xk}, \mathrm{n}$ and closing price of the next day is $\mathbf{X k},(\mathbf{n}+\mathbf{1}) \ldots . . . \ldots . . . \mathbf{X k},(\mathbf{n}+\mathbf{6})$ will be the seventh day from the first day. The sum of all divided by number of days will give simple moving average $\mathbf{A}$. The natural $\log$ difference of the moving average of the day $\mathrm{Xk}, \mathrm{n}$ and the seventh day $\mathbf{X k},(\mathbf{n}+\mathbf{6})$, deducted from 1 is taken as the output for training. The formula for the same is as follows.....

$1-[\ln (\mathbf{A X k},(\mathbf{n}+\mathbf{6}))-\ln (\mathrm{AXk}, \mathrm{n})](2)$

\section{Neural Network Architecture}

A neural network is a massively parallel distributed processor made up of simple processing unit which has a natural propensity for storing experiential knowledge and making it available for use. (Simon Haykin, (1999). Neural networks has remarkable ability to derive meaning from complicated or imprecise data, can be used to extract patterns and detect trends that are too complex to be noticed by either humans or other computer techniques. A trained neural network can be thought of as an "expert" in the category of information it has been given to analyze.

Neural networks are composed of a number of interconnected simple processing elements called neurons or nodes. They operate in parallel and actually resemble the biological neurons. 
International Journal of Science and Research (IJSR)

ISSN (Online): 2319-7064

Index Copernicus Value (2013): 6.14 | Impact Factor (2014): 5.611

w

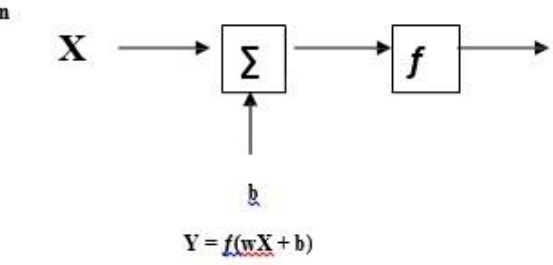

Figure 1: Transformation of Neurons

The neuron acts as a processing unit to transform the input to get an output. The neuron, like other linear or polynomial approximation, relates a set of input variables $\{\mathrm{Xi}\}, \mathrm{i}=1 \ldots \mathrm{k}$, to set of one or more output variables $\{Y i\}, i=1 \ldots k$. But in case of neural network the only difference is that it does not require any prior equation as in case of other approximation methods, rather the input variables are mapped to the output set by squashing or transforming by a special function known as activation function. Each neuron has a weight and a bias assigned to it. Each neuron receives an input signal, which transmits through a connection that multiplies its strength by the scalar weight $\mathrm{w}$, to form the product wX. A bias is added to the weighted input and is then passed through a transfer function to get the desired output. The weight $\mathrm{w}$ and the bias $b$ are the adjustable parameters of the neuron and are adjusted so that the neuron exhibits a desired behavior.

A neural network is a structure of two or more neurons in a layer with weighted connection between the neurons, which are often nonlinear scalar transformations.

The network shown below is a Multi-Layer Neural Network. The network architecture comprises of input layer with input neurons, one hidden layers with hidden neurons in the hidden layer and the output layer. The hidden layer of the neural network captures the data patterns and characteristics, and establishes a complex dynamic nonlinear relationship between the input and the output variable.

\section{Multi-Layer Neural Network}

The relationship between the input and the output of a neuron is established by the transfer function of the layer. The transfer function is a step function or a sigmoid function which takes the weighted input $\mathrm{n}$ and produces the output. Based on the performance of the network model transfer function are finalized for the network model.

The transfer functions used by us in the first layer is nonlinear and in the output layer linear function is used. The input layer function is also known as fire function.

The input function tested by us are log sigmoid function and zero-based log sigmoid function. The function used in the output layer is purelin or pure linear function.

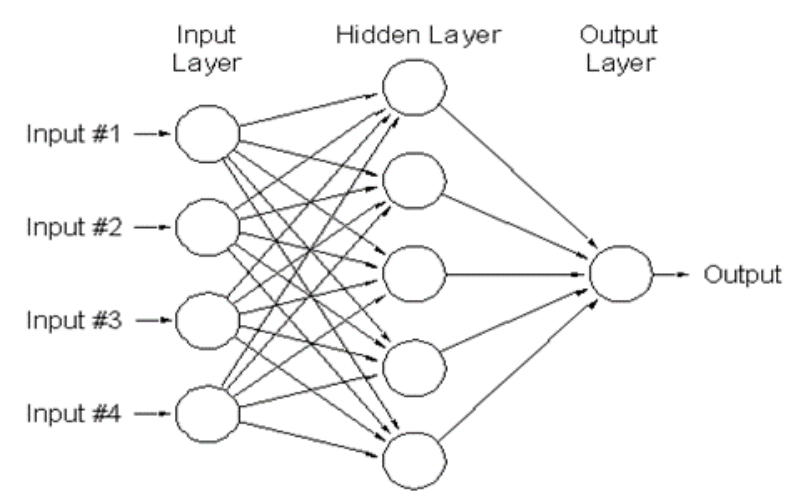

Figure 2: Multi-layer Neural Network

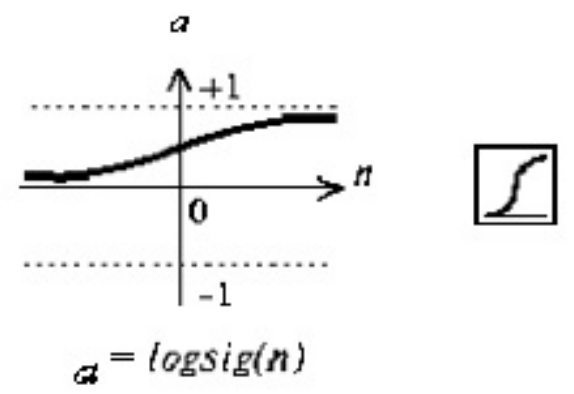

\section{Log-Sigmoid Transfer Function}

Figure 3: Log-Sigmoid Transfer Function

logsig is a transfer function. Transfer functions calculate a layer's output from its net input.

$\log \operatorname{sig}(\mathrm{N})$ takes one input, $\mathrm{N}-\mathrm{S} \times \mathrm{Q}$ matrix of net input (column) vectors and returns each element of $\mathrm{N}$ squashed between 0 and $1 . \log \operatorname{sig}(n)=1 /(1+\exp (-n))$

\section{Network Training}

After the neural network model is constructed, training of the neural network is the next essential step of the forecasting model. Training of neural network is an iterative process of nonlinear optimization of the parameters like weights and bias of the network. The result of the training process of the network depends on the algorithm used for the purpose. In this paper back propagation algorithm is used training. A back propagation network uses a supervised learning method for training.

In one complete cycle of the training process, a set of input data $\{X 1, X 2, X 3 \ldots\}$ is presented to the input node. The corresponding target output, is presented to the output node in order to show the network what type of behavior is expected. The output signal is compared with the desired response or target output and consequently an error signal is produced. In each step of iterative process, the error signal activates a control mechanism which applies a sequence of corrective adjustments of the weights and biases of the neuron. The corrective adjustments continue until the training data attains the desired mapping to obtain the target output as closely as possible. After a number of iterations the neural network is trained and the weights are saved. The test set of data is presented to the trained neural network to test the performance of the neural network. The result is recorded to see how well the net is able to predict the output using the adjusted weights of the network. 


\section{International Journal of Science and Research (IJSR) \\ ISSN (Online): 2319-7064}

Index Copernicus Value (2013): 6.14 | Impact Factor (2014): 5.611

Table 1: Following network structures are tested in this study:

\begin{tabular}{|c|c|c|c|c|}
\hline Network & Representation & $\begin{array}{c}\text { No of } \\
\text { Inputs }\end{array}$ & $\begin{array}{c}\text { Hidden } \\
\text { Neurons }\end{array}$ & Fire Function \\
\hline 1 & $10-10-1$ & 10 & 10 & Log Sigmoid \\
\hline 2 & $10-20-1$ & 10 & 20 & Log Sigmoid \\
\hline 3 & $20-10-1$ & 20 & 10 & Log Sigmoid \\
\hline 4 & $20-20-1$ & 20 & 20 & Log Sigmoid \\
\hline 5 & $10-10-1$ & 10 & 10 & $\begin{array}{c}\text { Zero Based } \\
\text { Log Sigmoid }\end{array}$ \\
\hline 6 & $10-20-1$ & 10 & 20 & $\begin{array}{c}\text { Zero Based } \\
\text { Log Sigmoid }\end{array}$ \\
\hline 7 & $20-10-1$ & 20 & 10 & $\begin{array}{c}\text { Zero Based } \\
\text { Log Sigmoid }\end{array}$ \\
\hline 8 & $20-20-1$ & 20 & 20 & $\begin{array}{c}\text { Zero Based } \\
\text { Log Sigmoid }\end{array}$ \\
\hline
\end{tabular}

Table 2: Performance

\begin{tabular}{|c|c|c|}
\hline Network & Performance(P) & Buy \& Hold Return \\
\hline 1 & $10.91 \%$ & $47.41 \%$ \\
\hline 2 & $14.36 \%$ & $47.41 \%$ \\
\hline 3 & $10.31 \%$ & $47.41 \%$ \\
\hline 4 & $11.87 \%$ & $47.41 \%$ \\
\hline 5 & $56.33 \%$ & $47.41 \%$ \\
\hline 6 & $57.06 \%$ & $47.41 \%$ \\
\hline 7 & $60.66 \%$ & $47.41 \%$ \\
\hline 8 & $70.42 \%$ & $47.41 \%$ \\
\hline
\end{tabular}

The returns are calculated as follows:

If $\mathbf{C t}$ is closing price of a trading session and $\mathbf{C t}+\mathbf{1}$ is the following trading session's close then performance $\mathbf{P}=$ $[(\mathbf{C t}+\mathbf{1}-\mathbf{C t}) * 100] / \mathbf{C t}$

All the structures explained above in table 1 are trained with 3000 epochs. From the results it is clear that the network 8 has been the most successful in forecasting the stock market. The buy and hold return during the tenure has been $-4.25 \%$ against which the network 8 has delivered $+30.42 \%$ return. The neural network returns are calculated considering a long position on buy signal and a short position on sell signal. The structure of the network has been 20 input variables with one hidden layer consisting of 20 hidden neurons and one linear output layer with one neuron. The fire function used in the network has been zero based log sigmoid function.

The second best structure has been network 5 with 10 input variables and one hidden layer with 10 neurons and one output layer with one linear neuron. Again the fire function zero based log sigmoid function has been used here. From the above mentioned results it is clear that zero based log sigmoid function has performed better then log sigmoid function.

\section{Future Scope of Improvement}

The neural network models for forecasting stock market are at an evolutionary stage and there are future possibilities of improvement in the prediction accuracy and reliability of the neural network based forecasting. In this paper I have investigated the basic aspect of the prediction problem of a stock market with artificial neural network. In this paper I have only used
Historic prices of the Index values for prediction. Other macro-economic factors and other international stock market data as input variables can also be used as input variables in order to improve the accuracy of the model. A further study should be carried out for a longer horizon with other network architectures. Application of Chaos Theory, Fractal analysis and wavelet analysis in feature selection of the input data set will also give a possibility of improvement in the performance. The various trend indicators of the technical analysis can also be used in the input variables and can be checked for improvement in the performance of the network. Furthermore a very basic model of ANN has been used in this study. By using various algorithms and combinations also a lot of improvement can be made. Further research is also required in the field of individual stock forecasting by ANN.

\section{Conclusion}

In this research I tried to create easy but useful neural network. Almost all the networks tested above are able to beat the buy and hold returns of the index. Couple of networks have outperformed the market by a significant margin. The return of $70.42 \%$ against $47.41 \%$ return of buy and hold by $20-20-1$ structure with zero based log sigmoid function is truly remarkable. The model has predicted the direction of seven day simple moving average after seven days.

In a highly volatile market like Indian Stock Market, the performance levels of the neural network models, reported in the paper will be very useful. Especially, the prediction of the direction of the market with fairly good accuracy will guide the investors and the regulators.

However, for prediction at different time in future, the network may be re-trained periodically and re-validated periodically with changes in the some of the features of the model. This is recommended as with time the characteristic and walk of the market changes and the network may miss out on the additional information.

I believe that neural network tool gives a promising direction to the study of prediction of the markets and other economic time series. The introduction of artificial intelligence will change the course of financial markets in future.

\section{References}

[1] Ritchie, J.C., 1996. Fundamental Analysis: a Backto-the-Basics Investment Guide to Selecting Quality Stocks. Irwin Professional Publishing

[2] Fama E, Schwert W. Asset returns and inflation. Journal of Financial Economics 1977;5:115-46.

[3] Keim D, Stambaugh R. Predicting returns in the stock and bond markets. Journal of Financial Economics 1986;17:357-90

[4] Qi, M., \& Wu, Y. (2003). Nonlinear prediction of exchange rates with monetary fundamentals. Journal of Empirical Finance, 10, 623-640. 


\section{International Journal of Science and Research (IJSR) \\ ISSN (Online): 2319-7064 \\ Index Copernicus Value (2013): 6.14 | Impact Factor (2014): 5.611}

[5] Saltoglu, B. (2003). Comparing forecasting ability of parametric and nonparametric methods: An application with Canadian monthly interest rates. Applied Financial Economics, 13, 169-176.

[6] Kanas, A., \& Yannopoulos1, A. (2001). Comparing linear and nonlinear forecasts for stock returns. International Review of Economics \& Finance, 10, 383-398.

[7] Kanas, A. (2001). Neural network linear forecasts for stock returns. International Journal of Finance \& Economics, 13, 317-354.

[8] Maasoumi, E., \& Racine, J. (2002). Entropy and predictability of stock market returns. Journal of Econometrics, 107, 291-312.

[9] Qi, M. (1999). Nonlinear predictability of stock returns using financial and economic variables. Journal of Business \& Economic Statistics, 17, 419429.

[10] Shively, P. A. (2003). The nonlinear dynamics of stock prices. Quarterly Review of Economics and Finance, 13, 505-517.

[11] Hamid , S. A., \& Iqbal, Z. (2004). Using neural networks for forecasting volatility of S\&P 500 Index futures prices. Journal of Business Research, 57, 11161125.

[12]Dunis, C. L., \& Huang, X. (2002). Forecasting and trading currency volatility: An application of recurrent neural regression and model combination. Journal of Forecasting, 13, 317-354.

[13] Jasic, T and wood, D.(2004)the profitability of daily stock market indices trades based on neural network predictions:case study for the s\&p 500. the day. the TOPIX and the FTSE in the period 1965-1999 applied financial economics, 14,285-297.

[14] Yao, J., Li, Y., \& Tan , C. L. (2000). Option price forecasting using neural networks. Omega, 28, 455466.

[15] Malliaris, M., \& Salchenberger, L. (1996). Using neural networks to forecast the S\& P 100 implied volatility. Neurocomputing, 10, 183-195.

[16] Srinivasan, N., Ravichandran, V., Chan, K.L., Vidhya, J.R., Ramakirishnan, S., Krishnan, S.M.; Exponentiated backpropagation algorithm for multilayer feedforward neural networks; Neural Information Processing, 2002. ICONIP apos; 02. Proceedings of the 9th International Conference, Volume 1, Issue, 18-22 Nov. 2002 Page(s): 327 - 331 vol.1

[17] Tsang, E.P.K., Markose, S. \& Er, H., Chance discovery in stock index option and future arbitrage, New Mathematics and Natural Computation, World Scientific, Vo.1, No.3, 2005, 435-447.

[18] Tsang, E.P.K., Computational intelligence determines effective rationality, International Journal on Automation and Control, Vol.5, No.1, January 2008, 63-66.

[19] Abhyankar, A., Copeland, L. S., \& Wong, W. (1997). Uncovering nonlinear structure in real-time stockmarket indexes: The S\&P 500, the DAX, the Nikkei 225, and the FTSE-100. Journal of Business \& Economic Statistics, 15, 1-14.

[20]Desai, V. S., \& Bharati, R., (1998). A comparison of linear regression and neural network methods for predicting excess returns on large stocks. Journal of Operations Research, 78, 127-163.

[21] Fernandez-Rodriguez, F., Gonzalez-Martel, C., \& Sosvilla-Rivero, S. (2000). On the profitability of technical trading rules based on artificial neural networks: Evidence from the Madrid stock market. Economic Letters, 69, 89-94.

[22] Goutam Dutta, Pankaj Jha, Arnab Kumar Laha and Neeraj Mohan Artificial Neural Network Models for Forecasting Stock Price Index in the Bombay Stock Exchange Journal of Emerging Market Finance,Vol. 5, No. 3, 283-295.

[23] Jung-Hua Wang; Jia-Yann Leu Stock market trend prediction using ARIMA-based neural networks Neural Networks, 1996., IEEE International Conference on Volume 4, Issue , 3-6 Jun 1996 Page(s):2160 - 2165 vol.4.

[24] Kim, Kyoung-Jae. Artificial neural networks with feature transformation based on domain knowledge for the prediction of stock index futures Intelligent Systems in Accounting, Finance \& Management, Vol. 12, Issue 3 , Pages $167-176$.

[25]Kim, S, K., and S.H Chun (1998) "Graded Forecasting Using An Array Of Bipolar Predictions: Application Of Probabilistic Neural Network To A Stock Market Index." International Journal of Forecasting, 14, 323-337.

[26] Leigh, W.Paz, M., \& Purvis, R. (2002). An analysis of a hybrid neural network and pattern recognition technique for predicting short-term increases in the NYSE composite index. OmegaInternational Journal of Management Science, 30, 6976.

[27] Pan Heping,Tilakaratne C and Yearwood John(2005): "Predicting Australian Stock Market Index using Neural Networks Exploiting Dynamic Swings and Inter-market Influences" Journal of Research and Practice in Information Technology, Vol 37. 1.

[28] García-Almanza, A.L. \& Tsang, E.P.K., Detection of stock price movements using chance discovery and genetic programming, International Journal of Knowledgebased and Intelligent Engineering Systems, Vol.11, No.5, December 2007, 329-344.

[29] Trippi, R.R., J.K. Lee (1996). Artificial Intelligence in Finance\&Investing: State-of-the- Art Technologies for Securities Selection and Portfolio Management. Irwin, Chicago.

[30]Charalambous, C. (1992). Conjugate gradient algorithm for efficient training of artificial neural networks. IEEE Proceedings, 139(3), 301-310.

[31]Coats, P., L. Fant (1990). Recognizing financial distress patterns using a neural network tool. Financial Management, Nov., 142-55.

[32] Stornetta, W.S., B.A. Huberman (1988). An improved three layered backprop algorithm. In M. Caudill and C. Butler (Eds.) Proceedings of the IEEE First International Conference on Neural Networks. San Diego, CA, SOS Printing.

[33] Majumdar, Hussian(2010). Forecasting of Indian Stock Market Index Using Artificial Neural Network. Research. WWW.NSEINDIA.COM 